\title{
Operative stabilization of flail chest injuries: review of literature and fixation options
}

\author{
D. C. Fitzpatrick · P. J. Denard · D. Phelan • \\ W. B. Long $\cdot$ S. M. Madey $\cdot$ M. Bottlang
}

Received: 5 April 2010/Accepted: 26 April 2010/Published online: 3 June 2010

(C) The Author(s) 2010. This article is published with open access at Springerlink.com

\begin{abstract}
Background Flail chest injuries cause significant morbidity, especially in multiply injured patients. Standard treatment is typically focused on the underlying lung injury and involves pain control and positive pressure ventilation. Several studies suggest improved short- and long-term outcomes following operative stabilization of the flail segments. Despite these studies, flail chest fixation remains a largely underutilized procedure.

Methods This article reviews the relevant literature concerning flail chest fixation and describes the different implants and techniques available for fixation. Additionally, an illustrative case example is provided for description of the surgical approach.

Results Two prospective randomized studies, five comparative studies, and a number of case series documented benefits of operative treatment of flail chest injuries, including a decreased in ventilation duration, ICU stay, rates of pneumonia, mortality, residual chest wall deformity, and total cost of care. Historically, rib fractures have been stabilized with external plates or intramedullary implants. The use of contemporary, anatomically contoured rib plates reduced the need for intraoperative plate
\end{abstract}

D. C. Fitzpatrick

Slocum Center for Orthopedics and Sports Medicine,

Eugene, OR, USA

\section{P. J. Denard}

Department of Orthopedics, Oregon Health and Science

University, Portland, OR, USA

D. Phelan · W. B. Long · S. M. Madey · M. Bottlang ( $ه)$

Biomechanics Laboratory, Legacy Clinical Research and

Technology Center, 1225 NE 2nd Avenue, Portland,

OR 97215, USA

e-mail: mbottlan@lhs.org bending. Intramedullary rib splints allowed less-invasive fixation of posterior fractures where access for plating was limited.

Conclusion Operative treatment can provide substantial benefits to patients with flail chest injuries and respiratory compromise requiring mechanical ventilation. The use of anatomically contoured rib plates and intramedullary splints greatly simplifies the procedure of flail chest fixation.

Keywords Flail chest - Rib fracture .

Surgical stabilization $\cdot$ Rib plate $\cdot$ Rib splint

\section{Introduction}

Flail chest injuries carried a dismal prognosis prior to the development of modern medical interventions. In the first half of the 1900s, management of these severe thoracic injuries included non-operative treatments such as external taping, patient positioning and traction by way of towel clips attached to the flail segment $[1,2]$. These techniques limited paradoxical motion at the fracture site but required the patient to remain supine for prolonged periods of time, which often led to more serious complications.

Several reports describing internal fixation of flail chest injuries appeared in the 1950s. Common surgical techniques included simple wire suture fixation of the fracture ends and rush rod fixation of the flail segment $[3,4]$. In the late 1950s the medical treatment of the underlying lung injury by 'internal splinting' of flail chest segments using positive pressure ventilation became popular [5]. The use of positive pressure ventilation resulted in improved patient outcomes relative to early non-operative and operative fixation [5]. However, this technique requires prolonged 
time on the ventilator, resulting in secondary chest infections and persistently high mortality rates of $10-36 \%$ in some series [6-9]. Additionally, positive pressure ventilation is not always able to reduce and stabilize the bony injury, resulting in painful fracture nonunion or symptomatic chest wall deformity in $64 \%$ of patients in one study [7]. In contrast, operative repair of severe chest wall injury is associated with low long-term morbidity and pain [10]. These factors led a number of surgeons to advocate for primary internal fixation of severe flail chest injuries. A small number of reports were published beginning in the 1960s with generally improved results relative to nonsurgical historical control groups. More recently, two comparative studies noted a reduction in mortality of 38 and $78 \%$ due to surgical stabilization $[11,12]$.

Despite these seemingly superior results, operative fixation of flail chest injuries remains an underutilized intervention [13]. This underutilization is secondary to multiple factors including the lack of familiarity with the few comparative studies of operative and non-operative treatment, surgical specialization in which fracture surgeons are not comfortable operating in the chest and thoracic surgeons are not familiar with modern fracture fixation principles, and the lack of appropriate fixation hardware to simplify the surgery and optimize outcomes. Mayberry et al. [14] surveyed a series of 405 cardiothoracic, trauma, and orthopedic surgeons concerning their views on chest wall fixation. They found that most surgeons felt chest wall fixation was indicated in some cases, but that very few had experience using the technique. They also found that most surgeons were unfamiliar with the literature concerning chest wall fixation and that there was an unfamiliarity of fixation techniques and instrumentation by the surgeons who typically operate on the chest. This article attempts to address these two issues by distilling the modern literature regarding flail chest fixation as well as reviewing the fixation options for stabilization of flail chest segments. Finally, our preferred technique of fixation is presented with an illustrative case.

\section{Literature review}

There are two level one studies comparing operative and non-operative treatment of flail chest injuries [11, 12]. Tanaka et al. [12] selected 37 patients out of 148 with flail chest who met strict inclusion criteria for a prospective, randomized study of surgical versus internal pneumatic stabilization. All patients required mechanical ventilation and had six or more consecutive rib fractures. All patients were initially treated with positive pressure ventilation. At 5 days post-injury, 18 patients were randomized to fixation with Judet struts and 19 patients continued with positive pressure mechanical ventilation. Only fractures occurring between ribs 4 and 10 were stabilized. Patients in each group had identical extubation criteria. They found significant differences in the number of days requiring mechanical ventilation (10.8 vs. 18.3), number of days in the ICU (16.5 vs. 26.8 ), and pneumonia ( $22 \%$ vs. $90 \%$ ) between the surgical and non-surgical groups, respectively. They also found a difference in the rate of return to work at 6 months $(61 \%$ vs. $5 \%)$ and the total cost of care $(\$ 13,455$ vs. $\$ 23,423)$. Patients in the non-surgical group had greater complaints of chest tightness, thoracic pain and dyspnea at 6 and 12 months after surgery.

A second randomized, prospective study by Granetzny et al. [11] compared outcomes in 20 patients treated using surgical fixation with intramedullary $\mathrm{k}$-wires and 20 patients treated non-surgically with adhesive plaster strapping. They found a significant difference in the number of days of ventilator use ( 2 vs. 12), the number of days in the ICU (9.6 vs. 14.6), residual chest wall deformity (1 vs. 9), pneumonia (10\% vs. $50 \%)$ and wound infection rates $(10 \%$ vs. $0 \%$ ) between the operative and non-operative groups, respectively. They also noted significantly improved pulmonary function tests performed at 2 months post-injury in the operative group.

Lardinois et al. [8] prospectively evaluated 66 patients who required surgery out of 732 total patients with a flail chest injury. All patients had anterolateral flail segments involving greater than 4 ribs. Indications for fixation included respiratory failure $(n=28)$, progressive displacement of the flail segment $(n=15)$, failure to wean for pulmonary reasons $(n=21)$ and thoracotomy due to associated injuries $(n=2)$. Pulmonary contusion involving on average $30 \%$ of the lung volume was present in $80 \%$ of the patients. Fixation was performed using stainless steel $3.5 \mathrm{~mm}$ reconstruction plates. They found that immediate extubation was possible in $47 \%$ of patients, and the mean ventilator time was 2.1 days. They also found a return to work rate at 2 months post-surgery of $100 \%$. Plate removal was required in $11 \%$ of patients because of hardware related pain.

Many experts believe that the underlying lung injury, rather than the bony injury, is the major contributor to the morbidity and mortality following flail chest injuries. Voggenreiter et al. [15] attempted to address this issue by retrospectively comparing the outcomes of surgical fixation of the flail segment in 10 patients with and 10 patients without underlying pulmonary contusion. They also evaluated 18 matched patients without pulmonary contusion treated non-surgically. Pulmonary contusion was diagnosed on bronchoscopy. Inclusion criteria were flail chest with the need for thoracotomy $(n=6)$, flail chest and respiratory failure without pulmonary contusion $(n=9)$, paradoxical chest wall motion $(n=3)$ and severe deformity of 
the chest wall $(n=2)$. Fractures were fixed with Judet struts or $3.5 \mathrm{~mm}$ plates. The results were focused on shortterm outcomes, namely the number of ventilator days, pneumonia and mortality. They found significant differences in the number of ventilator days (30.8 vs. 6.5$)$ and rates of pneumonia (40\% vs. $10 \%)$ and mortality (30\% vs. $0 \%$ ) between the surgical patients with and without pulmonary contusion. They also found significant differences in ventilator days (6.5 vs. 26.7$)$ and pneumonia (10\% vs. $27 \%$ ) between the surgical and non-surgical patients without pulmonary contusion. They suggested that patients with flail chest and no contusion have better outcomes if surgical stabilization is performed early, while patients with flail chest and pulmonary contusion should be fixed only if paradoxical motion or progressive collapse is noted.

Nirula et al. [16] retrospectively compared 30 flail chest patients treated surgically with 30 non-surgical patients matched base on age, Injury Severity Score and chest Abbreviated Injury Score. Indications for surgery included severe flail chest, pain, bleeding and failure to wean. They used Adkins Struts and wires to fix the flail segments. The primary outcome measures of ICU days and total hospital days did not show a statistically significant difference between the groups. However, the number of ventilator days measured from the time of surgery to extubation was significantly lower in the operative group (2.9) relative to the non-operative group (9.4).

Ahmed and Mohyuddin [17] compared 26 patients treated surgically with $\mathrm{k}$-wire fixation of one rib of the flail segment with 38 patients treated non-surgically using positive pressure ventilation. The rib was stabilized 'onretreat' after other surgical interventions including treatment of a hemothorax, treatment of a major air leak, or associated abdominal and orthopedic injuries. No statistical analysis was performed and the groups were not matched or randomized. They reported improved outcomes in the number of ICU days (21 vs. 9), the number of ventilator days (15 vs. 3.9), the number of patients requiring tracheotomy (37\% vs. $11 \%)$, the rate of chest infection $(50 \%$ vs. $15 \%)$, sepsis rate ( $24 \%$ vs. $4 \%$ ) and the mortality rate ( $29 \%$ vs. $8 \%$ ) between the non-operative and operative groups, respectively.

A number of case series studies have also been published which support the trends seen in these five comparative and two prospective studies [13, 18-21]. In general, there is a decrease in the number of days requiring mechanical ventilation, number of days in the ICU, and the rate of chest infection, all favoring operative fixation of the flail segment.

Nirula et al. [22] collected information on 650 rib fracture repairs reported in the literature to determine the complication rates associated with surgical fixation. Complications included eight superficial wound infections
(1.2\%), four draining wounds without infection, two pleural empyemas, one wound hematoma, one persistent pleural effusion and one case of osteomyelitis. They noted eight hardware failures and nine patients requiring hardware removal.

It is important to also understand that the inclusion criteria for these studies were fairly strict and that not all flail chest patients require surgical treatment. Common inclusion criteria were flail segments of 3-4 ribs or greater with one of the following associated conditions: failure to wean for pulmonary reasons, massive chest wall deformity with paradoxical chest wall motion, and respiratory failure despite the use of adequate peridural anesthesia. More controversial indications included fixation of rib fractures 'on retreat' from surgery for associated injuries, pain without respiratory failure and fixation in the setting of a pulmonary contusion without paradoxical motion or massive deformity.

\section{Implants}

\section{Stabilization with plates}

Over the past three decades, a considerable number of specialized rib plates have been introduced, emphasizing the persistent desire for operative stabilization of the chest wall (Fig. 1). Early rib plates were applied with sutures or circlage wires. In 1972, Paris et al. [23] introduced rib struts that were up to $40 \mathrm{~cm}$ long to span and suspend flail rib segments. These struts were applied along the rib, between ribs or across ribs using sutures, and were routinely removed upon fracture healing. Vecsei et al. [24] introduced a low-profile plate for circlage fixation that did not require routine removal. Because the flat cross-sectional profile of $1 \mathrm{~mm} \times 14 \mathrm{~mm}$ precluded in-plane contouring, these plates were only available up to a length of $8 \mathrm{~cm}$ and did not allow bridging of a flail segment. A subsequent generation of rib plates utilized claws that were tightened to the rib with forceps to simplify plate fixation and to reduce the risk of injury to intercostal neurovascular structures posed by circumferential suture fixation [19, 25]. The Judet plate (1973) had clawed end sections and a flat central section of $5.3 \mathrm{~cm}$ length suitable for spanning a single fracture $[25,26]$. The Labitzke claw plate was the first rib plate specifically designed to accommodate inplane bending [27]. It was sufficiently flexible to closely conform to the conical surface over long rib sections. While the Labitzke plate could span both fractures of a flail rib segment, its high flexibility necessarily limited the ability to rigidly suspend a flail segment. Stating the fundamental need for rigid spanning of a flail segment, Sanches-Lloret introduced $13-19 \mathrm{~cm}$ long rib struts with 


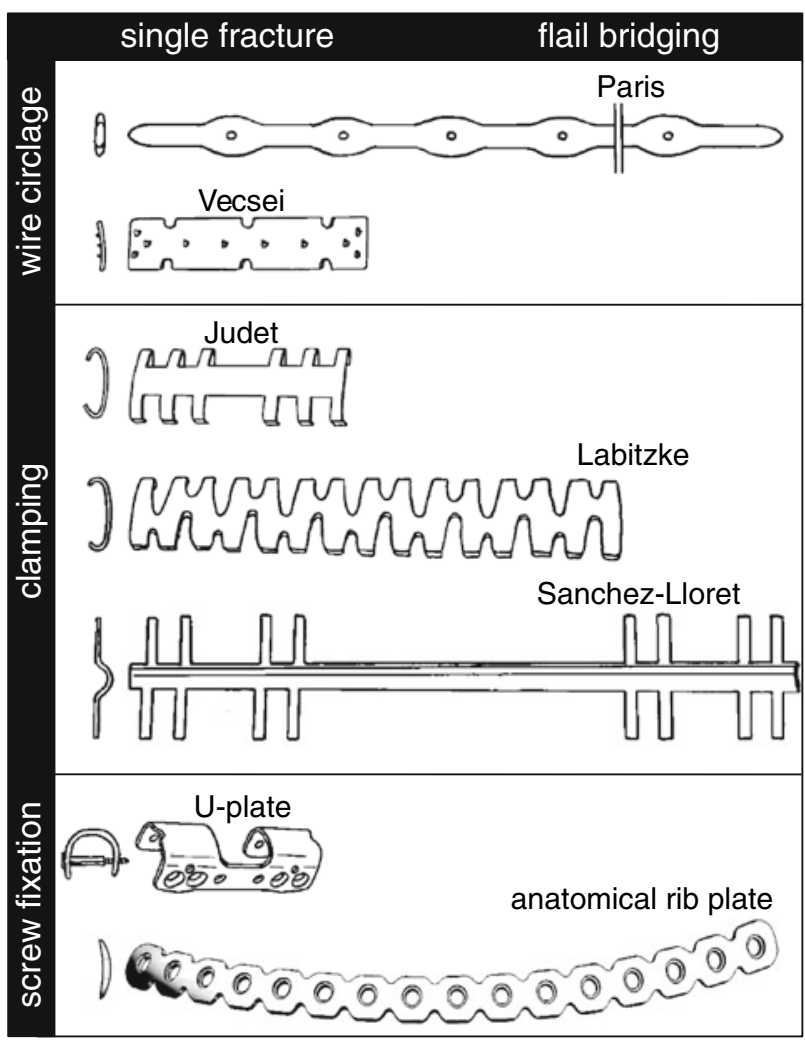

Fig. 1 Evolution of rib plates (drawings to scale in cross-sectional and frontal view): early plates were applied with sutures and circlage wires. To simplify plate application, a subsequent plate generation had "claws" that allowed clamping of the plate onto the rib surface. Contemporary rib plates are applied with screws that securely lock into the plate. Rib plates can be categorized into short implants for fixation of a single fracture site (Vecsei et al. [24], Judet [26], U-plate [29]), and long implants that also permit bridging and suspension of a flail segment (Paris et al. [23], Labitzke [19], Sanchez-Lloret et al. [28]). Anatomical rib plates [32] made of flexible titanium can be used for bridging fixation of multiple fractures or can be shortened as needed for fixation of a single fracture

clawed end sections to bridge fractures on each side of the flail rib segment with a single implant [28]. The circular mid-section of the struts accommodated contouring but lacked the low profile of plates. For minimally invasive fixation of a single fracture, Sales et al. [29] recently introduced a U-plate with a length of less than $5 \mathrm{~cm}$.

Despite introduction of specialized plates, standard $1 / 3$ tubular and $3.5 \mathrm{~mm}$ reconstruction plates remain the most frequently used plates for rib fracture fixation, likely because of their broad availability. These standard plates accommodate in-plane contouring and provide sufficient stability to suspend a flail segment. However, these plates can be too stiff and may cause stress concentrations that contribute to screw pullout in osteoporotic ribs $[9,15,27$, 30]. Furthermore, Labizke [19] stated that these plates require bending and twisting, making their application more technically demanding and more time-intensive

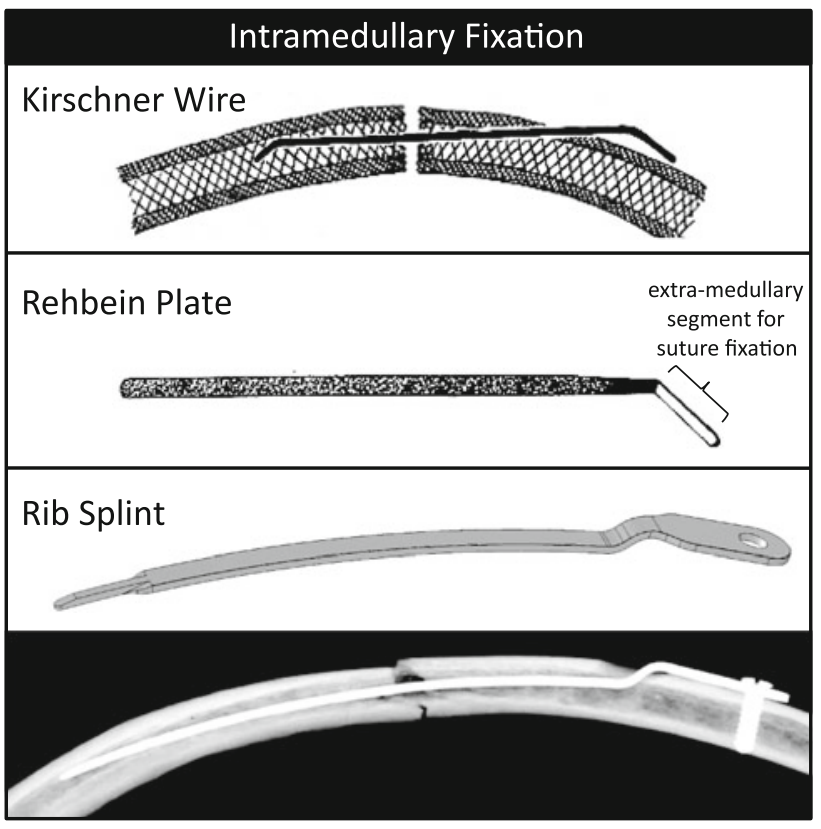

Fig. 2 Intramedullary fixation of rib fractures with Kirschner wires [34], Rehbein plates [40], and contemporary rib splints [38, 39]

compared to flexible claw plates. For plate contouring of standard plates, Oyarzun et al. [31] recommended that the rib fracture should be reduced first, a template should be contoured to the rib surface, and a plate should be contoured to the template using bending irons, pliers, or a bending press. This complexity frequently required a team approach, whereby the general/thoracic surgeon provided the operative exposure and collaborated with the orthopaedic service for plate fixation [13]. More recently, anatomic rib plates were introduced with the goal of reducing or eliminating the need for templating and pre-bending of the plate [32]. By their anatomically contoured nature, they also facilitate direct reduction of the rib fragments to the plate. This can reduce surgical complexity and time, especially when multiple ribs require stabilization, as in the case of a flail chest. Specific to flail chest stabilization, anatomic rib plates support the use of long plates that enable strong yet low-profile bridging and suspension of a flail segment.

\section{Intramedullary stabilization}

Intramedullary implants have also been used as splints to hold the flail segment in a more anatomic position and prevent paradoxical motion without obtaining rigid fixation (Fig. 2). Intramedullary fixation has several benefits relative to plate fixation, including less surgical dissection required for insertion and less prominent hardware, which limits the need for removal at a later time. Kirschner wire (k-wire) fixation of ribs is reported in multiple studies over 
Fig. 3 a CT scan performed immediately post injury showing two levels of displaced rib fractures with volume loss relative to the contralateral hemithroax. b Post-operative CT scanogram showing the four rib plates on the anterolateral rib fractures and the four splints used to stabilize the posterior fractures. The use of splints for the posterior fractures allows relative stability of the fractures without the need for excessive dissection
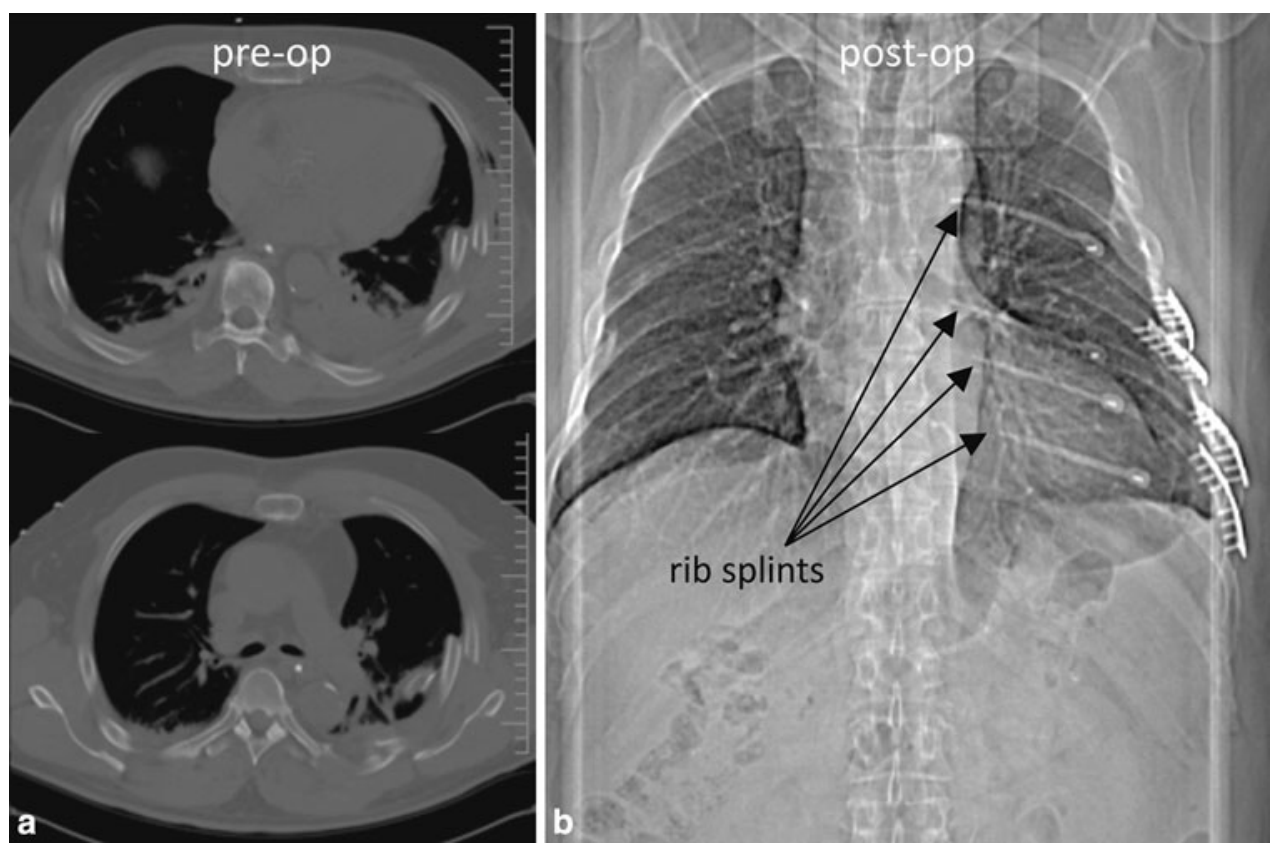

the past 50 years with generally good results $[9,17,33$, 34]. Authors have noted rotational instability of the fractures, caused by the small circular cross section of the k-wires [30, 33]. Another reported problem with intramedullary k-wire fixation is the potential for loss of fracture reduction with migration of the wire resulting in pain or additional injury to the surrounding tissues [17, 25, 35, 36]. The Rehbein plate, an intramedullary plate with a rectangular cross section designed to provide improved rotational stability was introduced in 1972 [37]. One end of the plate was designed to be left out of the canal and was sutured to the rib to limit migration. More recently, a pre-contoured rib splint was introduced for intramedullary fixation of rib fractures [38]. Similar to the Rehbein plate, this splint has a rectangular cross section, but it is pre-contoured to fit the rib and is fixed to the rib with a locking screw to eliminate migration and to provide angular stability. Biomechanical analysis of this implant showed significant benefits relative to simple k-wire fixation [39]. Specifically, rib splint constructs were $48 \%$ stronger than Kirschner wire constructs. Rib splints furthermore prevented the complications of cut-out and migration seen with Kirschner wires. Therefore, rib splints are an attractive intramedullary solution for less-invasive stabilization of rib fractures, especially in case of posterior rib fractures where access for plating is limited.

\section{Case example}

The following case example is provided to illustrate rational indications for surgical fixation of rib fractures as well as the use of modern flail chest fixation techniques using newer anatomically contoured implants.

A 70-year-old man was admitted to the emergency ward following a high-speed motor vehicle collision. On evaluation in the emergency room he complained of chest pain but was hemodynamically stable with an oxygen saturation of $92 \%$ on room air. Physical examination revealed comfortable respirations without paradoxical chest wall motion. Radiographs and a computed tomography (CT) scan of the chest revealed segmental fractures of the left 3rd through 10th ribs with a hemopneumothorax (Fig. 3a). A chest tube was placed and he was admitted to the intensive care unit for observation. Associated injuries included right 3rd through 5th rib fractures, a sternal fracture, left renal laceration, grade I splenic laceration, a pelvic ring injury, and a T3 spinous process fracture. Total injury severity score was 21 .

On post-injury day (PID) 1 the patient developed progressive respiratory distress. He was unable to maintain adequate oxygenation and required mechanical ventilation. His pulmonary status continued to worsen and on PID four he required an increase in the $\mathrm{FIO}_{2}$ from 70 to 100 . Chest radiographs showed increased atelectasis of the left lower lobe. A volumetric chest CT was obtained which showed a left lung volume of $914 \mathrm{~cm}^{3}$ compared to $1,760 \mathrm{~cm}^{3}$ on the right. The increasing ventilatory requirements and decreased lung volume prompted the decision to stabilize the chest wall. On PID five an open reduction and internal fixation of the left 5 th through 9 th ribs was performed with the MatrixRIB $^{\mathrm{TM}}$ Fixation System (Synthes CMF, West Chester, PA). Intramedullary rib splints stabilized the posterior fractures of the 5th and 7 th through 9 th ribs (Fig. 3b). 

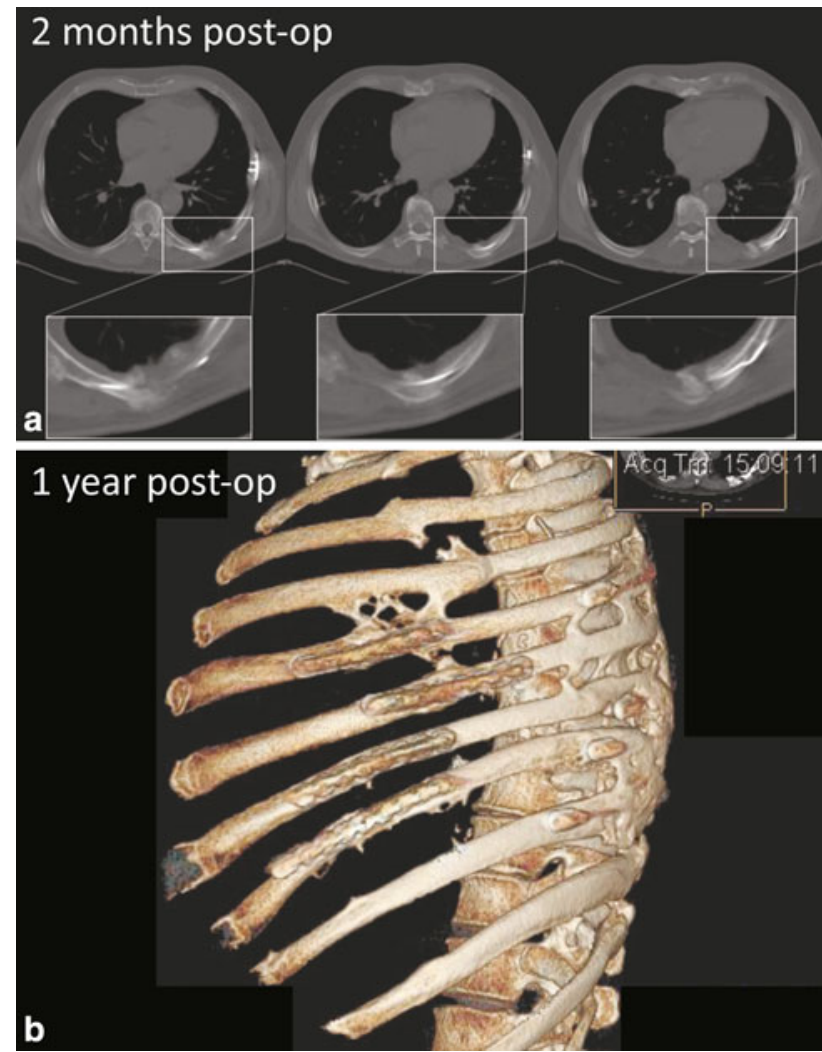

Fig. 4 a Two months postoperative CT focusing on one rib splint. There is abundant callus formation confirming healing of the fractures by means of secondary bone healing. The chest volume is returned to normal. b One year postoperative CT rendering

Anterolateral fractures of these ribs were secured using anatomical rib plates with three locking screws on each side of the fracture. No plate contouring was required. Operative time was $140 \mathrm{~min}$.

The patient was extubated on postoperative day seven and discharged 19 days after surgery. A 2-month postoperative CT scan showed intact hardware and fixation, as well as abundant formation of fracture healing callus at the fracture sites (Fig. 4a). At 3 months post-injury he required no narcotic pain medications and was swimming for rehabilitation. FVC and $\mathrm{FEV}_{1}$ were over $100 \%$ of predicted at 4.1 and $3.1 \mathrm{~L}$ respectively. Visual Analogue Scale scores were 0 for chest pain, 1.3 for chest tightness, and 0 for implant-related discomfort. A 1-year follow-up CT confirmed complete bony remodeling and intact hardware and hardware fixation (Fig. 4b).

\section{Conclusions}

The indications for fixation of flail chest injuries remain controversial mostly because of a lack of adequate studies comparing operative and non-operative treatment.
However, several studies suggest a substantial benefit to patients with flail chest injuries and respiratory compromise requiring mechanical ventilation. Despite this evidence, the procedure remains mostly underutilized. The goal of this work is to better inform the reader of the literature regarding chest wall fixation, given the fact that many surgeons are not aware of the published reports on flail chest fixation.

The use of anatomically contoured titanium plates and splints greatly simplifies the procedure of flail chest fixation. The pre-contoured plates are used for complex fractures and are long enough to allow bridging fixation of multiple fractures, including the entire flail segment, if desired. Because they are thin and manufactured from titanium, they are able to flex with respiration, limiting the risk of hardware or fixation failure secondary to repetitive loading. Additionally, the plates are low profile and prevent hardware irritation to minimize the need for removal after the fractures heal.

Rib splints are used for simple fractures, especially posterior fractures of the flail segment where access for plating is limited. Use of the splint for the posterior fractures reduces the need to elevate the latissimus dorsi, allowing fixation with less surgical dissection. Rib splints provide stable but flexible fixation of the fracture fragments. Use of a combination of plates and splints is common in our practice and allows the greatest possible combination of fixation options to fix these complex chest wall injuries.

Conflict of interest statement One or more of the authors receive consulting/royalty payments from Synthes CMF related to technology discussed.

Open Access This article is distributed under the terms of the Creative Commons Attribution Noncommercial License which permits any noncommercial use, distribution, and reproduction in any medium, provided the original author(s) and source are credited.

\section{References}

1. Cohen EA. Treatment of the flail chest by towel clip traction. Am J Surg. 1955;90:517-21.

2. Hudson TR, McElvenny RT, Head JR. Chest wall stabilization by soft tissue traction: a new method. JAMA. 1954;156:768-9.

3. Coleman FP, Coleman CL. Fracture of ribs: a logical treatment. Surg Gynecol Obstet. 1950;90(2):129-34.

4. Crutcher RR, Nolen TM. Multiple rib fracture with instability of chest wall. J Thorac Surg. 1956;32:15-21.

5. Avery EE, March ET, Benson DW. Critically crushed chest: a new method of treatment with continuous mechanical hyperventilation to produce alkalotic apnea and internal pneumatic stabilization. J Thoracic Surg. 1956;32:291-311.

6. Cacchione RN, Richardson JD, Seligson D. Painful nonunion of multiple rib fractures managed by operative stabilization. J Trauma. 2000;48(2):319-21. 
7. Landercasper J, Cogbill TH, Lindesmith LA. Long-term disability after flail chest injury. J Trauma. 1984;24(5):410-4.

8. Lardinois D, Krueger T, Dusmet M, Ghisletta N, Gugger M, Ris HB. Pulmonary function testing after operative stabilisation of the chest wall for flail chest. Eur J Cardiothorac Surg. 2001;20(3): 496-501.

9. Schmit-Neuerburg KP, Weiss H, Labitzke R. Indication for thoracotomy and chest wall stabilization. Injury. 1982;14(1):2634.

10. Mayberry JC, Kroeker AD, Ham LB, Mullins RJ, Trunkey DD. Long-term morbidity, pain, and disability after repair of severe chest wall injuries. Am Surg. 2009;75(5):389-94.

11. Granetzny A, Abd M, Emam E, Shalaby A, Boseila A. Surgical versus conservative treatment of flail chest. Evaluation of the pulmonary status. Interact Cardiovasc Thorac Surg. 2005;4(6): 583-7.

12. Tanaka H, Yukioka T, Yamaguti Y, Shimizu S, Goto H, Matsuda $\mathrm{H}$, Shimazaki S. Surgical stabilization of internal pneumatic stabilization? A prospective randomized study of management of severe flail chest patients. J Trauma. 2002;52(4):727-32.

13. Richardson JD, Franklin GA, Heffley S, Seligson D. Operative fixation of chest wall fractures: an underused procedure? Am Surg. 2007;73(6):591-6. discussion 596-597.

14. Mayberry JC, Ham LB, Schipper PH, Ellis TJ, Mullins RJ. Surveyed opinion of American trauma, orthopedic, and thoracic surgeons on rib and sternal fracture repair. J Trauma. 2009;66(3): 875-9.

15. Voggenreiter G, Neudeck F, Aufmkolk M, Obertacke U, SchmitNeuerburg KP. Operative chest wall stabilization in flail chestoutcomes of patients with or without pulmonary contusion. J Am Coll Surg. 1998;187(2):130-8.

16. Nirula R, Allen B, Layman R, Falimirski ME, Somberg LB. Rib fracture stabilization in patients sustaining blunt chest injury. Am Surg. 2006;72(4):307-9.

17. Ahmed Z, Mohyuddin Z. Management of flail chest injury: internal fixation versus endotracheal intubation and ventilation. J Thorac Cardiovasc Surg. 1995;110(6):1676-80.

18. Balci AE, Eren S, Cakir O, Eren MN. Open fixation in flail chest: review of 64 patients. Asian Cardiovasc Thorac Ann. 2004;12(1): $11-5$.

19. Labitzke R. Early thoracotomy and chest wall stabilization with elastic rib clamps. Zentralbl Chir. 1981;106(20):1351-9.

20. Mayberry JC, Terhes JT, Ellis TJ, Wanek S, Mullins RJ. Absorbable plates for rib fracture repair: preliminary experience. J Trauma. 2003;55(5):835-9.

21. Mouton W, Lardinois D, Furrer M, Regli B, Ris HB. Long-term follow-up of patients with operative stabilisation of a flail chest. Thorac Cardiovasc Surg. 1997;45(5):242-4.

22. Nirula R, Diaz JJ Jr, Trunkey DD, Mayberry JC. Rib fracture repair: indications, technical issues, and future directions. World J Surg. 2009;33(1):14-22.
23. Paris F, Tarazona V, Blasco E, Canto A, Casillas M, Pastor J, Paris M, Montero R. Surgical stabilization of traumatic flail chest. Thorax. 1975;30(5):521-7.

24. Vecsei V, Frenzel I, Plenk H Jr. A new rib plate for the stabilization of multiple rib fractures and thoracic wall fracture with paradoxical respiration. Hefte Unfallheilkd. 1979;138:279-82.

25. Menard A, Testart J, Philippe JM, Grise P. Treatment of flail chest with Judet's struts. J Thorac Cardiovasc Surg. 1983;86(2): $300-5$.

26. Judet R. Osteosynthese costale. Rev Chir Orthop Reparatrice Appar Mot. 1973;59(Suppl 1):334-5.

27. Labitzke R. Biomechanic examination of rib plates. Langenbecks Arch Chir. 1981;354(3):169-71.

28. Sanchez-Lloret J, Letang E, Mateu M, Callejas MA, Catalan M, Canalis E, Mestres CA. Indications and surgical treatment of the traumatic flail chest syndrome. An original technique. Thorac Cardiovasc Surg. 1982;30(5):294-7.

29. Sales JR, Ellis TJ, Gillard J, Liu Q, Chen JC, Ham B, Mayberry JC. Biomechanical testing of a novel, minimally invasive rib fracture plating system. J Trauma. 2008;64(5):1270-4.

30. Engel C, Krieg JC, Madey SM, Long WB, Bottlang M. Operative chest wall fixation with osteosynthesis plates. J Trauma. 2005; 58(1):181-6.

31. Oyarzun JR, Bush AP, McCormick JR, Bolanowski PJ. Use of 3.5-mm acetabular reconstruction plates for internal fixation of flail chest injuries. Ann Thorac Surg. 1998;65(5):1471-4.

32. Bottlang M, Helzel I, Long WB, Madey S. Anatomically contoured plates for fixation of rib fractures. J Trauma. 2010;68(3): $611-5$.

33. Meier P, Schupbach P. Therapy of the unstable thorax in serial fractures of the ribs. Schweiz Med Wochenschr. 1978;108(16): 608-13.

34. Moore BP. Operative stabilization of nonpenetrating chest injuries. J Thorac Cardiovasc Surg. 1975;70(4):619-30.

35. Albrecht F, Brug E. Stabilization of the flail chest with tension band wires of ribs and sternum. Zentralbl Chir. 1979;104(12): $770-6$.

36. Shah TJ. On internal fixation for flail chest. J Thorac Cardiovasc Surg. 1996;112(3):849-50.

37. Schupbach P, Meier P. Indications for the reconstruction of the unstable thorax due to serial rib fractures and respiratory insufficiency. Helv Chir Acta. 1976;43(5-6):497-502.

38. Helzel I, Long W, Fitzpatrick D, Madey S, Bottlang M. Evaluation of intramedullary rib splints for less-invasive stabilisation of rib fractures. Injury. 2009;40(10):1104-10.

39. Bottlang M, Helzel I, Long W, Fitzpatrick D, Madey S. Lessinvasive stabilization of rib fractures by intramedullary fixation: a biomechanical evaluation. J Trauma. 2010;68(5):1218-24.

40. Tscharner C, Schupbach P, Meier P, Nachbur B. Surgical treatment of the unstable thorax in respiratory insufficiency. Helv Chir Acta. 1989;55(5):711-7. 